\title{
QUALITY IMPROVEMENT OF A GEAR TRANSMISSION BY MEANS OF GENETIC ALGORITHM
}

doi: 10.2478/cqpi-2019-0052

Date of submission of the article to the Editor: 06/04/2019

Date of acceptance of the article by the Editor: 27/05/2019

Paweł Lempa ${ }^{1}$ - orcid id: 0000-0002-9421-440X

Edward Lisowski ${ }^{1}$ - orcid id: 0000-0002-7437-7391

Fumito Masui ${ }^{2}$ - orcid id: 0000-0001-9979-8734

Grzegorz Filo ${ }^{1}$ - orcid id: 0000-0003-0848-6124

Michal Ptaszynski² - orcid id: 0000-0002-1910-9183

Mariusz Domagała ${ }^{1}$ - orcid id: 0000-0001-9642-6142

Joanna Fabiś-Domagała ${ }^{1}$ - orcid id: 0000-0003-2811-1100

${ }^{1}$ Cracow University of Technology, Faculty of Mechanical Engineering, Jana Pawla II 37, Cracow, Poland

${ }^{2}$ Kitami Institute of Technology, Department of Computer Science, 165 Koen-cho Kitami, Hokkaido 090-8507, Japan

Abstract: The article deals with the issue of quality improvement of a gear transmission by optimizing its geometry with the use of genetic algorithms. The optimization method is focused on increasing productivity and efficiency of the pump and reducing its pulsation. The best results are tested on mathematical model and automatically modelled in 3D be means of PTC Creo Software. The developed solution proved to be an effective tool in the search for better results, which greatly improved parameters of pump especially reduced flow pulsation.

Keywords: optimization, genetic algorithm, gear transmision

\section{INTRODUCTION}

Gear pumps are among the most popular positive-displacement pumps used in industry. They are used in different range of pressures and flows. The advantage of this construction is a simple design that uses two gears as a positive-displacement components. They are usually gears with external teeth and outline involute. Selecting the right number of teeth, module, correction coefficients and other geometrical parameters is essential for the obtained operational parameters. The choice of design parameters of the pump requires compromise. For example, increasing the number of teeth reduces the pulsation of the pump, but it also reduces the delivery. Facilitating the selection of solutions the optimization problem can be formulated. Modelling the geometry of pumps and other hydraulic objects is realized by using parametric CAD systems. With appropriate list of parameters can automatically generate 3D models of 
various solutions. For this purpose, it is necessary to prepare the appropriate software that on the basis of the loaded parameters generates model. The article presents own software which, using different types of genetic algorithms, through API interface is connected to the CAD system and can automatically generate 3D models and carry out their quality improvement.

\section{MATHEMATICAL MODEL}

For the purpose of quality improvement of a gear transmission optimization can be carried out taking into account the different objective functions. In the case of positivedisplacement pumps it may be getting the biggest pump delivery or the total efficiency, minimize pulsation or cost of manufacture product, getting higher operating pressure etc.In the literature the most common approach is formulating one objective with a fixed range of decision variables formulating one objective with a fixed range of decision variables. However, it appears that better effects can be achieved by using multi-criteria optimizations. In this paper as a optimization objectives for the analyzed pump were selected: maximum unitary delivery, maximum pump delivery, minimum of pulsation and the total efficiency. Mathematical relationships for these functions can be determined from the following relationships:

GEOMETRIC DEPENDENCIES OF THE GEAR PUMP

Geometrical relationships can be described by the following formulas:

- $\quad$ proper delivery (unitary delivery)

$$
q=\pi b\left(\frac{d_{a}^{2}}{2}-\frac{d^{2}}{2}-2 k \frac{\left(\pi m \cos \left(\propto_{o}\right)\right)^{2}}{12}\right)
$$

where: Qu - unitary delivery, b -gearwheel width, $\mathrm{m}$ - module, $\mathrm{z}$ - number of teeth

- theoretical delivery

$$
Q_{t}=\frac{\pi b m^{2}}{2}\left(2\left(\frac{l_{z}}{2}+x+y\right)^{2}-\frac{l_{z}^{2}}{2} \frac{\cos \left(\alpha_{o}\right)^{2}}{\cos \left(\alpha_{w}\right)^{2}}-2 \frac{\pi^{2} \cos \left(\alpha_{o}\right)^{2}}{12}\right)
$$

where: $\alpha_{0}-$ nominal pressure angle, $\alpha_{w}$ - rolling pressure angle

- pulsation:

$$
\delta=\frac{Q_{\max }-Q_{\min }}{Q_{t}}
$$

where: $\delta$ - pulsation, $Q_{\max }$ - maximum pump delivery, $Q_{\min }$ - minimum pump delivery. The maximum and minimum delivery can be calculated from formulas:

$$
\begin{gathered}
Q_{\max }=\frac{b \omega_{1}}{2}\left(\frac{d_{a}^{2}}{2}-\frac{d^{2}}{2}\right) \\
Q_{\min }=\frac{b \omega_{1}}{2}\left(\frac{d_{a}^{2}}{2}-\frac{d^{2}}{2}-2 \frac{\left(\pi m \cos \left(\propto_{o}\right)\right)^{2}}{4}\right)
\end{gathered}
$$

continuing transformation:

$$
\begin{gathered}
Q_{\max }=\pi b n\left(\frac{d_{a}^{2}}{2}-\frac{d^{2}}{2}\right) \\
Q_{\min }=\pi b n\left(\frac{d_{a}^{2}}{2}-\frac{d^{2}}{2}-2 \frac{\left(\pi m \cos \left(\propto_{o}\right)\right)^{2}}{4}\right)
\end{gathered}
$$

Substituting equation (6) and (7) to formula the pulse (3), we obtain:

$$
\delta_{e w}=\frac{2 \pi^{2} \cos \left(\alpha_{o}\right)^{2}}{4\left(2\left(\frac{l_{z}}{2}+x+y\right)^{2}-\frac{l_{z}^{2} \cos \left(\alpha_{o}\right)^{2}}{22 \cos \left(\alpha_{w}\right)^{2}}-\frac{\pi^{2} \cos \left(\alpha_{o}\right)^{2}}{6}\right)}
$$

THE EFFICIENCY OF THE PUMP

The parameters associated with the operation of the gear pump are: fluid parameters, working pressure, and the total pump efficiency, which is defined as the product of the 
mechanical, hydraulic and volumetric efficiency. Total efficiency $\eta_{c}$ is defined as the quotient of output power $\mathrm{P}_{\text {out }}$ to the power input $\mathrm{P}_{\text {in }}$ or it can be calculated as the product of the volumetric efficiency $\eta_{\mathrm{v}}$ and hydraulic-mechanical efficiency $\eta_{\mathrm{hm}}$ :

$$
\eta_{c}=\frac{P_{\text {out }}}{P_{\text {in }}}=\eta_{v} \eta_{h m}
$$

Volumetric efficiency is defined as the quotient of real delivery Qr to a theoretical delivery Qt:

$$
\eta_{v}=\frac{Q_{r}}{Q_{t}}=\frac{Q_{t}-\Delta Q}{Q_{t}}=1-\frac{\Delta Q}{Q_{t}}
$$

where: $\Delta Q$ - volumetric losses.

$$
\Delta Q=Q_{\mu}+Q_{\varrho}
$$

where: $Q_{\mu}$ - losses associated with viscosity of the liquid, $Q_{\rho}$ - losses associated with the density of the liquid.

$$
Q_{\mu}=c_{\mu} \frac{p}{2 \pi \mu_{d}} q
$$

where: $\mu_{d}$ - dynamic viscosity of liquid, $c_{\mu}$ - loss coefficient associated with the viscosity, depending on the unitary delivery.

$$
Q_{\varrho}=c_{r} \sqrt{\frac{2 p}{\varrho}} \sqrt[3]{q^{2}}
$$

where: $\rho$ - density of the liquid, $c_{r}$ - loss coefficient associated with the density, depending on the type and size of the slots, and the unitary delivery.

Substituting relations (11), (12), (13) for (10) receives a formula which defines the volumetric efficiency:

$$
\eta_{v}=1-c_{\mu} \frac{p}{2 \pi \mu_{d} n}-c_{r} \frac{1}{n} \sqrt{\frac{2 p}{\varrho}} \sqrt[3]{q^{-1}}
$$

Hydraulic-mechanical efficiency is defined as the quotient of the theoretical moment $\mathrm{M}_{\mathrm{t}}$ on the pump shaft to the actual moment $\mathrm{M}_{\mathrm{r}}$ :

$$
\eta_{h m}=\frac{M_{t}}{M_{r}}=\frac{M_{t}}{M_{t}+\Delta M}
$$

where $\Delta \mathrm{M}$ is a moment of loss.

$$
M=M_{v}+M_{Q}+M_{p}
$$

where: $\mathrm{M}_{\mathrm{v}}$ - moment of losses associated with the resistances caused by the friction of viscous liquids, $\mathrm{M}_{\rho}$ - moment of losses associated with the density of the working medium, $\mathrm{M}_{\mathrm{p}}$ - moment of losses associated with the mechanical losses dependent on the working pressure of the pump.

The moments can be calculated from the following formulas:

$$
\begin{gathered}
M_{v}=c_{v} \mu_{d} n q \\
M_{\varrho}=c_{\varrho} \frac{n^{2}}{4 \pi} \sqrt[3]{q^{5}} \\
M_{p}=c_{p} \frac{p q}{2 \pi}
\end{gathered}
$$

where: $c_{v}$ - proportionality coefficient, $c_{\varrho}$ - density coefficient, $c_{p}$ - pressure coefficient. Substituting dependences (16), (17), (18) and (19) for (15) we receives a formula defining the hydraulic-mechanical efficiency:

$$
\eta_{h m}=\frac{1}{1+c_{v} 2 \pi \frac{\mu_{d}{ }^{n}}{p}+c_{p} \frac{\varrho n^{2} 3}{2 \pi} \sqrt[3]{q^{2}}+c_{p}}
$$


Substituting the formula for volumetric efficiency (14) and model of the hydraulicmechanical efficiency (20) we get the following formula on the total efficiency:

$$
\eta_{c}=\frac{1-c_{\mu} \frac{p}{2 \pi \mu_{d} n}-c_{r} \frac{1}{n} \sqrt{\frac{2 p}{\varrho}} \sqrt[3]{q^{-1}}}{1+c_{v} 2 \pi \frac{\mu_{d} n}{p}+c_{\varrho} \frac{\varrho n^{2}}{2 p} \sqrt[3]{q^{2}}+c_{p}}
$$

On basis of following formulas (1), (2), (8), (21) constituting mathematical model of the pump, four objective functions were built. The following notations were used: $\mathrm{f}_{1}$ - function of the unitary delivery (22), $\mathrm{f}_{2}$ - function of the theoretical delivery (23), $\mathrm{f}_{3}$ - function of the pulsation, (24) (for compatibility with other functions, i.e. maximum $f_{3}$ means the minimum pulsation), $\mathrm{f}_{4}$ - function of the total efficiency (39).

$$
\begin{gathered}
f_{1}=f_{1}\left(q\left(b, r_{w}, r_{t}\right)\right) \\
f_{2}=f_{2}\left(Q_{t}(x, y, z, b)\right) \\
f_{3}=-1 f_{3}(\delta(z, y, z, b)) \\
f_{4}=f_{4}\left(\eta_{c}\left(Q_{e w}, p, \mu_{d}, \varrho\right)\right)
\end{gathered}
$$

For the defined objective function multi-criteria optimization problem can be written as:

$$
f=\max \left(f_{1}, f_{2}, f_{3}, f_{4}\right)
$$

\section{GENETIC ALGORITHM APPROACH AND SIMULATIONS}

Genetic Algorithms are subset of Evolutionary Algorithms (EAs), and they were inspired by the process of natural selection known from genetics. Bio-inspired operators Ared used in them for selection, crossover and mutation to generate optimized solutions. Genetic Algorithms represent randomized heuristic search strategies that simulates natural selection process, where the population is composed of possible solutions. They are focused on evolving a population from which strong and diverse candidates can emerge via mutation and crossover (mating). Different types of Genetic Algorithms were already introduced by different researchers, moreover the same type of GA can bring different quality of solutions, depending on multiple variables, which include starting population, number of generations or fitness function. Finding the best starting parameters and type of GA the most appropriate for a given optimization problem is a next challenge. For that reason, specific library that automatically applies multiple types of GAs in optimization purposes was created and used.

For that library $\mathrm{C}++$ language was used as main language. In first step of experiments were used basic functions of library that allowed the use of several different kinds of GAs like: Simple GA, Uniform Crossover GA, n-point Crossover GA, GA with sexual selection, GA with chromosome aging and so forth. Different sets of parameters for GA including were prepared, such as: population size, starting population, number of generations, type of mutation and crossover. In the second stage of experiments advanced functions of the library as using of multithreaded processing for running several GAs in the same time were used.

Calculations were carried out for various variants of the pump design and for different values of pressure and oil viscosity. To carry out the calculations a hydraulic oil with the following specifications was selected: kinematic viscosity of the fluid $\mu \mathrm{k}=41 \mathrm{~mm} 2=\mathrm{s}$, density $\rho=840 \mathrm{~kg}=\mathrm{m} 3$, and the module of gearwheel is $\mathrm{m}=3: 5$. There were also used a decision variables as: correction coefficient $(x)$, number of teeth $\left(I_{z}\right)$, height tooth coefficient $(y)$, gearwheel width (b) and the pumping pressure ( $p)$. All of this 
values were also included in experimentation with different genetic algorithms. There were two main approaches in experiments for quality improvement of a gear transmission - to use a predefined sets of the ranges for decision variables with different genetic algorithms and starting parameters, where sets were defined based on knowledge from other researchers and second approach -to let algorithms define the decision variables ranges completely by themselves. In comparison of that two approaches the final values were really similar, but second approach usually took much more generations get to final results. Also sets of values finally used in second approach were usually much narrower than predefined sets based on literature and experience, but it was possible only after running many experiments with similar starting parameters genetic algorithms.

For the purpose of defining starting parameters of used genetic algorithms, were prepared Simple Genetic Algorithm, Uniform Crossover Genetic Algorithm, 2-point, 3point and 5-point Crossover Genetic Algorithm with and without limited lifespan and Genetic algorithm with Sexual reproduction wit and without limited lifespan. Population was mixed initialized, with bigger weight on randomization. For stop conditions were used three approaches: Max number of generation set by user or chosen from predefined sets (like 100,300,500, 1000, 2000, etc.), max number of generation without improvement and minimal objective function value achieved. For parents selection were used Fitness Proportional selection, Roulette selection, Tournament selection, Ranking selection and Sexual selection. For mutations experiment has been conducted for Bit Flip Mutation, Random Resetting, Swap Mutation, Scramble Mutation and Inversion Mutation. New population generation with different percentage value used three different types: Replacing the whole old generation, Fitness Based Selection, Age Based Selection. Also influence of different size of starting population was tested. So big amount of experiments with many different variables, tested separately and with correlation to each other was possible thanks to use of multithreading, with different parameters for algorithms running in the same time.

One example of the biggest effect of carried out optimization was reduction of the pump pulsation by $57.9 \%$ (Figure 1), total efficiency improved by $12.3 \%$ and proper delivery also slightly improved by $3.4 \%$.

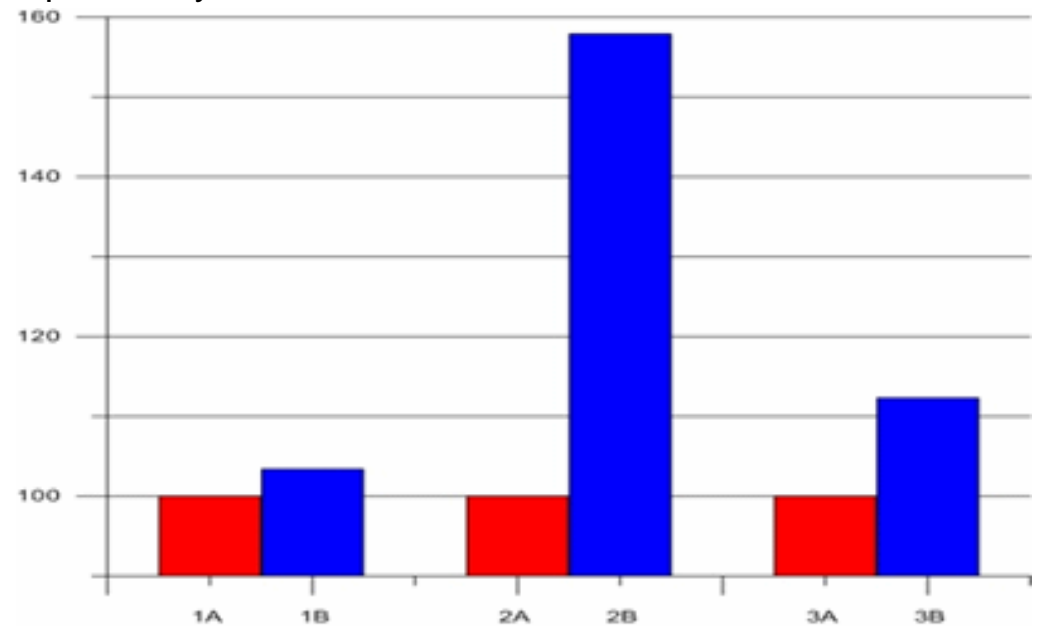

Figure 1 Comparison of the pump before optimization - $A$ and after optimization - B:

1 - proper delivery, 2 - pulsation, 3 - total efficiency

Fig. 2 shows example of simplified 3D model generated by software after generating on of most optimal results. 


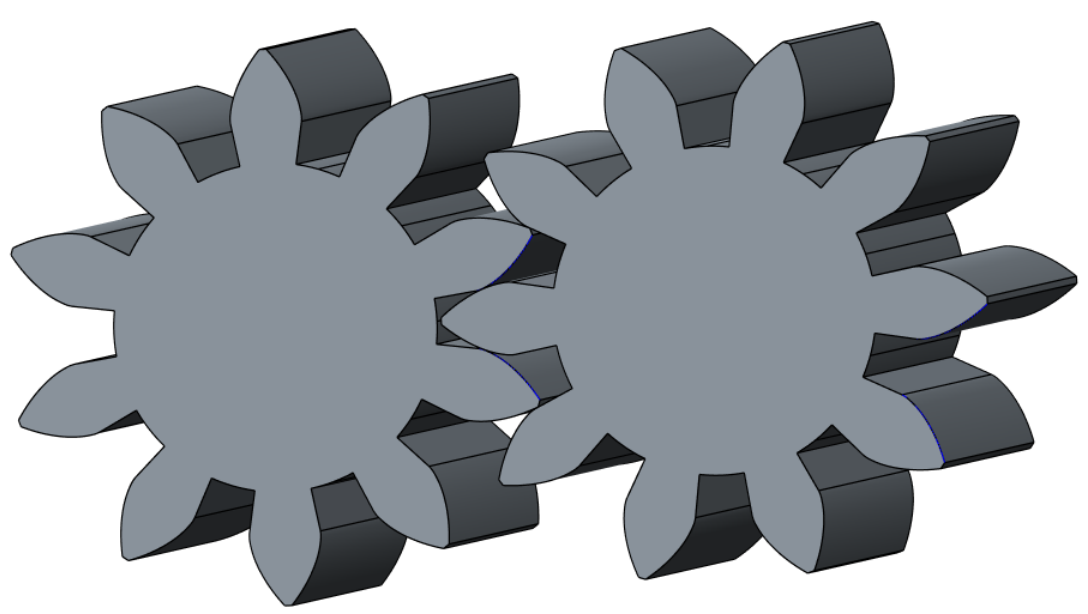

Fig. 2. Example of simplified 3D model generated by software

\section{SUMMARY}

This paper presents the problem of multi-criteria optimization of gear transmission with the use of a different types of genetic algorithms. For this purpose proprietary software that can automatically build a 3D model in the program Creo was created. Quality improvement of was carried out by using a gear pump known in the literature mathematical models describing the basic properties of the pump, such as proper delivery, theoretical delivery, pulsation and efficiency. As an object of study selected standard gear pump solution used in industry. On it, simulation studies were carried out in search for better solutions to reduce pulsation, improve theoretical and unitary delivery and total efficiency. To accomplish this task, used the program Creo (Pro/Engineer) for the purpose of construction of the gearwheels geometry and developed own software for optimization with the use of genetic algorithm. The developed software proved to be an effective tool in the search for better solutions, which greatly improved parameters of pump especially reduced pump pulsation.

The methodology presented in this paper may be useful in other areas of research and development. Stochastic optimization, especially with quick convergence, may be very powerful tool in those situations, where constraints are vague, optimization criterion is defined by a recipe, not a math formulation, and the dimensionality of the decision space is very large. It may a typical management problem (Kozien and Kozien, 2017; Kielbus and Karpisz, 2019), but also a research investigation (Radek et al., 2014; Augustyn and Kozien, 2014; Opydo et al., 2016; Szczotok et al., 2018) or development (Karpisz and Kielbus, 2018; Pacana and Pacana, 2018; Radek et al., 2018) with many factors. This approach can be particularly useful in numerical experiments when the object under study is a mathematical model with high complexity. It leads to construct a surrogate model (Pietraszek and Goroshko, 2014) with lower complexity but with higher uncertainty (Pietraszek et al., 2017) which quantification requires Monte-Carlo based methods (Gadek-Moszczak et al., 2015).

\section{REFERENCES}

Augustyn, E.; Kozien, M. S. 2014. A Study on Possibility to Apply Piezoelectric Actuators for Active Reduction of Torsional Beams Vibrations. Acta Physica Polonica A, 125, A164-A168. 
El-Mahdy, O., Ahmed, M., Metwalli, S., 2010. Computer aided optimization of natural gas pipe networks using genetic algorithm. Applied Soft Computing, 10, 1141-50. DOI: 10.1016/j.asoc.2010.05.010

Frith, R., Scott, W., 1996. Comparison of an external gear pump wear model with test data. Wear, 196, 64-71. DOI: 10.1016/0043-1648(95)06845-7

Fuh, J., Li, W., 2005. Advances in collaborative CAD: the-state-of the art. Computer Aided Design, 37, 571 -81. DOI: 10.1016/j.cad.2004.08.005.

Gadek-Moszczak, A., Pietraszek, J., Jasiewicz, B., Sikorska, S., Wojnar, L., 2015. The bootstrap approach to the comparison of two methods applied to the evaluation of the growth index in the analysis of the digital $x$-ray image of a bone regenerate. New Trends in Comp. Coll. Intell., 572, 127-136. DOI: 10.1007/978-3-319-10774-5_12

Gen, M., Cheng, R., 2000. Genetic algorithms and engineering optimization Vol. 7. Wiley, Hoboken.

Goldberg, D.E., Holland, J.H., 1988. Genetic algorithms and machine learning. Machine Learning, 3, 95-9.

Grefenstette, J.J., 1986. Optimization of control parameters for genetic algorithms. IEEE Transactions on Systems, Man and Cybernetics, 16, 122-8.

Hu, Z.H., Ding, Y.S., Zhang, W.B., Yan, Q., 2008. An interactive co-evolutionary CAD system for garment pattern design. Computer Aided Design, 40, 1094-104. DOI: 10.1016/j.cad.2008.10.010

Ionel, I.I., 1986. Pumps and pumping. Elsevier, New York.

Ivantysyn, J., Ivantysynova, M., 2003. Hydrostatic pumps and motors: principles, design, performance, modelling, analysis, control and testing. Tech Books International.

Karpisz, D., Kielbus, A., 2018. Selected problems of designing modern industrial databases. MATEC Web Conf., 183, art. 01017. DOI: 10.1051/matecconf/201818301017

Kielbus, A., Karpisz, D., 2019. Risk management as a process security tool. System Safety: Human-Technical Facility-Environment, 1, 234-239. DOI: 10.2478/czoto2019-0030

Kita, E., Tanie, H., 1997. Shape optimization of continuum structures by genetic algorithm and boundary element method. Engineering Analysis with Boundary Elements, 19, 129-36. DOI: 10.1016/S0955-7997(97)00014-3

Kollek W. Pompy zebate - konstrukcja i eksploatacja. Zakład Narodowy im. Ossolinskich; 1996.

Kozien, E., Kozien, M.S., 2017. Academic governance as a determinant of efficient management of a university in Poland - legal and comparative perspective. ESD 2017: Economic and Social Development Conf., Madrid, Varazdin, 38-47.

Krenich, S., 2017. Multi-thread evolutionary computation for design optimization. Technical Transactions, 9, 197-206

Lampinen J., 2003. CAM shape optimisation by genetic algorithm. Computer Aided Design, 35, 727-37. DOI: 10.1016/S0010-4485(03)00004-6

Ladd, S.R., 1995. Genetic algorithms in C++. Hungry Minds, New York.

Langdon, W.B., Poli, R., 2002. Foundations of genetic programming. Springer.

Melanie, M., 1999. An introduction to genetic algorithms. Cambridge, Massachusetts.

Opydo, M., Kobylecki, R., Dudek, A., Bis, Z. 2016. The effect of biomass co-combustion in a CFB boiler on solids accumulation on surfaces of P91 steel tube samples. Biomass \& Bioenergy, 85, 61-68. DOI: 10.1016/j.biombioe.2015.12.011 
Osmera, P., Lacko, B., Peter M., 2003. Parallel Evolutionary Algorithms, 2003 IEEE Int. Symposium Computational Intelligence in Robotics and Automation, Kobe, IEEE, 1348-1353.

Pacana, J., Pacana, A., 2018. Analysis of Possibilities of Using Polymeric Materials for Testing Prototypes of Harmonic Drive. Materials Research Proceedings, 5, 61-66. DOI: 10.21741/9781945291814-11

Pal, P., Tigga, A., Kumar, A., 2005. Feature extraction from large cad databases using genetic algorithm. Computer Aided Design, 37, 545-58. DOl: 10.1016/j.cad.2004.08.002

Park, H.S., Dang, X.P., 2010. Structural optimization based on CAD-CAE integration and metamodeling techniques. Computer-Aided Design, 42, 889-902. DOI: 10.1016/j.cad.2010.06.003.

Pietraszek, J., Dwornicka, R., Krawczyk, M., Kołomycki, M., 2017. The non-parametric approach to the quantification of the uncertainty in the design of experiments modelling. UNCECOMP 2017: $2^{\text {nd }}$ Int. Conf. Uncertainty Quantification in Computational Sciences and Engineering, Rhodes, NTU of Athens, 598-604. DOI: 10.7712/120217.5395.17225

Pietraszek, J., Goroshko, A., 2014. The heuristic approach to the selection of experimental design, model and valid pre-processing transformation of DoE outcome. Advanced Materials Research-Switzerland, 874, 145-149. DOI: 10.4028/www.scientific.net/AMR.874.145

Radek, N., Pasieczynski, L., Makrenek, M., Dudek, A., 2018. Mechanical Properties of Anti-Graffiti Coating Systems used in the Railway Industry. Materials Research Proceedings, 5, 243-247. DOI: 10.21741/9781945291814-43

Radek, N., Pietraszek, J., Antoszewski, B., 2014. The Average Friction Coefficient of Laser Textured Surfaces of Silicon Carbide Identified by RSM Methodology. Adv. Mat. Res.-Switz., 874, 29-34. DOI: 10.4028/www.scientific.net/AMR.874.29

Shi, X., 2011. Design optimization of insulation usage and space conditioning load using energy simulation and genetic algorithm. Energy, 36, 1659-67. DOI: 10.1016/j.energy.2010.12.064

Stroustrup, B., 2000. The C++ Programming Language. The C++ Programming Language (Special Edition). Addison-Wesley, Reading.

Szczotok, A., Radek, N., Dwornicka, R., 2018. Effect of the induction hardening on microstructures of the selected steels. METAL 2018: $27^{\text {th }}$ Int. Conf. Metallurgy and Materials. Ostrava, Tanger, 1264-1269.

Wang, N., Tai, K., 2010. Target matching problems and an adaptive constraint strategy for multiobjective design optimization using genetic algorithms. Computers and Structures, 88, 1064-73. DOI: 10.1016/j.compstruc.2010.06.002

Wang, D., Zhang, W., Yang, J., Wang, Z., 2012. A virtual punching method for shape optimization of openings on curved panels using CAD-based boolean operations. Computer Aided Design, 44, 388-99. DOI: 10.1016/j.cad.2012.01.003

Wang, N.F., Tai, K., 2010. Target matching problems and an adaptive constraint strategy for multiobjective design optimization using genetic algorithms. Computers and Structures, 88, 1064-1073. DOI: 10.1016/j.compstruc.2010.06.002 